\title{
Blumengrüße aus Kenia - zu welchem Preis? Das kenianische Arbeitsrecht und seine Umsetzung auf den
Blumenplantagen
}

\author{
Von Katrin Merhof, Konstanz*
}

\section{A. Einleitung}

„Wir produzieren, was wir nicht essen - wir essen, was wir nicht produzieren“, zitiert „Die Zeit" den protestantischen Kirchenführer Tolbert Jallah aus Liberia. ${ }^{1}$ Das trifft auf viele afrikanische Länder zu, denen die Hilfsgelder für die ländliche Entwicklung gekürzt wurden und die keine Chance hatten, auf dem Markt der subventionierten Lebensmittel preislich mitzuhalten. ${ }^{2}$ Doch dieses Interesse verfolgten die Akteure in der Entwicklungspolitik, allen voran die internationalen Finanzinstitute, auch gar nicht. Stattdessen setzte man auf andere Wirtschaftszweige - in Kenia beispielsweise auf die Blumenindustrie. ${ }^{3}$ Diese geht dort in die 1980er Jahre zurück und wuchs seitdem signifikant. So wurden 1990 noch 14.000 Tonnen Schnittblumen exportiert - im Jahr 2008 waren es 93.000 Tonnen. ${ }^{4}$ Die Bedeutung dieses Industriezweigs zeigt sich auch im Zusammenhang der Menschen, deren Existenz davon abhängt: Etwa 500000 Kenianer arbeiten direkt und zwischen 50000 und 60000 indirekt in der Blumenindustrie. ${ }^{5}$ Exporte nach Deutschland spielen dabei eine wichtige Rolle. Von Januar bis November 2008 sind frische Schnittblumen im Wert von 682,4 Millionen Euro nach Deutschland eingeführt worden; nach den Niederlanden war Kenia (18,3 Millionen Euro) dabei der wichtigste Lieferant. ${ }^{6}$ Beschäftigt auf den Blumenplantagen sind vorwiegend Frauen: Zum einen haben sie in aller Regel eine schlechtere Schulbildung als Männer und sind daher auf Beschäftigungsmöglichkeiten im Niedriglohnsektor beschränkt. Zum anderen werden sie geschätzt wegen ihrer ,zarten Hände“ und ihrer

* Kathrin Merhof, Jura-Studium und Referendariat in Freiburg, Barcelona, Berlin und Phnom Penh. Doktorandin an der Justus-Liebig-Universität Gießen (Prof. Dr. Thilo Marauhn). E-Mail: katrin.merhof@gmx.de.

1 Grefe, Christiane: Heute schon gegessen? In: Die Zeit Nr. 47 vom 12.11.2009, Politik S. 8.

2 Grefe (o. Fn. 1) S. 8.

3 Wanjiru Gichure, Christine: The common good in global business - the missing factor in "ethical sourcing"? Abrufbar unter www.globethics.net/web/isbee-collection/collection-articles (13.07.2010). S. 2.

4 Kenya Flower Council: Market Data. www.kenyaflowercouncil.org/marketdata.php (13.07.2010).

5 Kenya Flower Council (o. Fn. 4).

6 Statistisches Bundesamt Deutschland: Zum Valentinstag: Rosen aus Amsterdam. 
billigen Arbeitskraft. ${ }^{7}$ Weil in deutschen Supermärkten ein Strauß Rosen schon für wenige Euro erstanden werden kann, drängt sich die Frage auf, wie diese Ware trotz aufwändiger Aufzucht und eines langen Transportwegs so preiswert verkauft werden kann. Die Antwort liegt auf der Hand: Wie bei so vielen anderen Waren - etwa Kaffee, Bananen, Zucker, Baumwolle, um nur einige zu nennen - tragen die Arbeitskräfte ${ }^{8}$ in den Ursprungsländern die Last, um uns Europäern den billigen Blumenkauf zu ermöglichen. Was für Rechte und vor allem: was für Rechtsverletzungen - stehen dahinter? Dieser Frage soll hier nachgegangen werden.

\section{B. Die individualarbeitsrechtliche Situation}

\section{Der Vertragsschluss}

Schon der Vertragsschluss kann ein erstes Hindernis für das Rechtsbewusstsein und die Rechtswahrnehmung der Arbeiterinnen darstellen. Ein Vertrag kann nach Artikel 8 des Employment Act 2007 (EA) mündlich und schriftlich geschlossen werden. Ein Arbeitsvertrag über mehr als drei Monate oder über ein Werk, dessen Herstellung erwartungsgemäß mehr als drei Monate in Anspruch nehmen wird, soll schriftlich geschlossen werden, Artikel 9 Abs. 1 (b) EA. Kommt es zu gerichtlichen Auseinandersetzungen und kann der Arbeitgeber einen schriftlichen Vertrag nicht vorlegen, obwohl er einen solchen hätte abschließen müssen, so obliegt ihm die Beweislast für eine Vertragsklausel, Artikel 10 Abs. 7 EA.

Im Wesentlichen werden diese gesetzlichen Vorgaben eingehalten. Sowohl dauerhaft als auch befristet angestellten Arbeitskräfte gaben in einer Studie des Natural Resources Institute (NRI), die von Wissenschaftlerinnen der Northeastern University, der University of Nairobi und dem Institute of Development Studies durchgeführt wurde, an, sie hätten einen schriftlichen Vertrag bekommen, den beide Parteien unterzeichnet hätten und in dem die vertraglichen Rechte und Pflichten aufgelistet gewesen seien. Gelegenheitsarbeiter ${ }^{9}$ dagegen erhielten eigenen Angaben zufolge nur mündliche Verträge, was aber in Einklang mit der Gesetzeslage steht. Allerdings gibt es keine Norm, die vorschreibt, in welcher Sprache ein Vertrag abgefasst werden muss, was sich in einem Land mit zwei Amtssprachen und weiteren über 50 Sprachen verschiedener Volksgruppen nicht von selbst ergibt. In der Blumenindustrie sind die Verträge in der Regel in Englisch verfasst - und werden von den

Organisation für eine solidarische Welt: Valentinstag 2007 - "Fairschenkt Rosen", www.oew. org/de/presseartikel.php?id=753 (13.07.2010).

Im Folgenden steht der Begriff "Arbeitskräfte" für Arbeiterinnen und Arbeiter (wobei aber beachtet werden muss, dass Frauen ca. 70\% der Arbeitskraft in der Blumenindustrie ausmachen). In den Bereichen, in denen nur Frauen betroffen sind, wird selbstverständlich der Begriff der Arbeiterin verwendet.

9 Gelegenheitsarbeiter sind solche Arbeiter, die am Ende eines jeden Tages bezahlt werden und nicht länger als 24 aufeinander folgende Stunden angestellt sind (Artikel 2 EA). Mehr dazu unter B. IV. 
Arbeitern daher häufig nicht verstanden. ${ }^{10}$ Schließlich handelt es sich zum größten Teil um ungelernte Arbeitskräfte mit nur geringer Schulbildung, und auch wenn Englisch Amtssprache ist, so sprechen doch gerade auf dem Land die meisten Kenianer Swahili oder eine der anderen zahlreichen Stammessprachen. Schon bei der Arbeitsaufnahme treten also die ersten Schwierigkeiten für die Arbeiter in der Blumenindustrie auf, um ihre Rechte wahrzunehmen. Weitere Probleme begegnen ihnen aber auch in vielen anderen Bereichen.

\section{Lohn und Unterkunft}

Das kenianische Arbeitsrecht regelt detailliert, wann und auf welche Art und Weise der Lohn zu bezahlen ist (Artikel $17 \mathrm{EA}$ ), wann er fällig wird (Artikel $18 \mathrm{EA}$ ) und die Umstände, unter denen der Arbeitgeber eine Lohnkürzung vornehmen darf (Artikel 19 EA). Vor allem aber Kritik ausgesetzt ist die Regelung der Höhe des Lohns, die sich nicht im Employment Act befindet, sondern in Verordnungen für verschiedene Wirtschaftszweige. Art. 3 der VO zur Regulierung der Löhne in der Landwirtschaft ${ }^{11}$ (The Regulations of Wages [Agricultural Industry] Order RWO) untersagt Vergütungen unterhalb des gesetzliche Mindestlohns.

Da es keine nationale Regelung des Mindestlohns gibt, kann der Arbeitsminister einen Mindestlohn für bestimmte Arbeitergruppen oder Sparten festlegen (Artikel 46 des Labour Institutions Act 2007 (LIA)). Zuvor muss er den von ihm eingerichteten Rat über die Löhne in der Landwirtschaft und das „National Labour Board“ anhören, eine Kommission aus Arbeitgeber- und Arbeitnehmervertretern, die den Minister berät (Artikel 43 Abs. 1 b), 46 Abs. 1 LIA). ${ }^{12}$ Der ministerielle Lohnvorschlag ist im Amtsblatt zu veröffentlichen und hat Gelegenheit zur Stellungnahme zu geben, ehe er endgültig festlegt wird (Artikel 45 LIA). Den Mindestlohn in der Landwirtschaft regelt eine Ergänzung des RWO ${ }^{13}$ Eine ungelernte Arbeitskraft erhält einen monatlichen Mindestlohn von 3043 Schillingen, ${ }^{14}$ etwa 29,00 EUR. Pro Tag müssen mindestens 127,75 Schillinge gezahlt werden, also etwa 1,20 Euro.

Nach allen Studien halten sich die Arbeitergeber im Großen und Ganzen an den gesetzlichen Mindestlohn bzw. zahlen mitunter sogar mehr. ${ }^{15}$ Das Problem ist aber, dass selbst

Catherine Dolan / Maggie Opondo / Sally Smith: Gender, Rights \& Participation in the Kenya Cut Flower Industry. 2002. www.nri.org/projects/NRET/kenyareportfinal2.pdf (13.07.2010). S. 34.

Die Blumenindustrie gehört nach Art. 2 Abs. 1 a) des Agriculture Act zur Landwirtschaft.

Dies gilt nur für die Festlegung der Löhne in der Blumenindustrie. Für die Festlegung von Löhnen im nicht-landwirtschaftlichen Bereich muss der Rat für die allgemeine Lohnfestsetzung angehört werden (General Wages Council), Artikel 43 Abs. 1 a) LIA.

Regulations of Wages (Agricultural Industry) (Amendment) Order 2009.

Für andere Gruppen wie etwa Nachtwächter, Köche, Vorarbeiter, Traktorfahrer fallen höhere Mindestlöhne zwischen 3514 und 4458 Schillingen an. Dies betrifft allerdings nur einen kleinen Teil der Arbeiter auf den Blumenplantagen.

Dolan/Opondo/Smith (o. Fn. 10) S. 9. 
der Mindestlohn nicht zur Existenzsicherung einer Arbeiterin und ihrer Familie ausreicht ${ }^{16}$ : Eine Studie von 2008 bezifferte den Grundversorgungsbedarf für eine fünfköpfige Familie in zwei Wochen auf 3500 Schillinge, Kleidung o.ä. unberücksichtigt. ${ }^{17}$ Hinzu kommt, dass in der Regel von einem Arbeitseinkommen mehrere Verwandte versorgt werden müssen. Bei der von der Organisation „Kenya Women Workers“ (KEWWO) durchgeführten Studie, bei der 150 Arbeitskräfte von 15 Blumenfirmen befragt wurden, gaben $45 \%$ an, von ihrem Lohn hingen fünf bis zehn Personen ab; bei über $5 \%$ waren es sogar mehr als zehn Personen. ${ }^{18}$ So wird meist versucht, mit einem weiteren zweiten Job oder Arbeit im informellen Sektor, im Ackerbau oder durch die Zuwendungen besser situierter Angehöriger das Familieneinkommen aufzubessern. ${ }^{19}$ Mitunter ist dies aber auch verboten, so etwa durch die 6000 Menschen beschäftigende Blumenfirma „Sher Agencies“, die ihren Mitarbeitern jegliche Nebentätigkeit untersagt. ${ }^{20}$

Natürlich lässt sich das Einkommen auch durch Aufstieg erhöhen. Da die Blumenindustrie aber nur wenige Arbeitsplätze kennt, die eine höhere Ausbildung oder längere Arbeitserfahrung benötigen, gibt es auch nur selten Beförderungen. ${ }^{21}$ Hier wäre also das Handeln der Regierung in Form einer Erhöhnung des Mindestlohns gefragt. Allerdings gibt es in Kenia dieselben Diskussionen um die Wirkungen eines Mindestlohns wie in Deutschland. So droht die Blumenindustrie beispielsweise, bei einer Erhöhung des Mindestlohns ihre Standorte zu wechseln. Da sich inzwischen Nachbarländer wie Tansania, Äthiopien und Uganda im Geschäft mit den Schnittblumen betätigen, verfängt diese Drohung zumeist. Davon abgesehen leidet auch die Blumenindustrie enorm unter der Weltwirtschaftskrise: So ist der Blumenexport im ersten Quartal 2009 um ein Drittel eingebrochen $^{22}$; der Umsatz am Valentinstag 2009 war der schlechteste in der Geschichte der kenianischen Blumenindustrie ${ }^{23}$. Als Folge haben die Vertreter der Blumenindustrie angekündigt, Kosten zu sparen, wo es eben geht, um Plantagenschließungen - die es schon gab - zu

16

17 vom 19.04.2009. www.theeastafrican.co.ke/news/-/2558/561646/-/rigsbaz/- (13.07.2010).

International Federation of Human Rights in cooperation with the Centre for Research on Multinational Cooperation: Economic Development or Human Rights? Assessing the impact of Kenya's Trade and Investment Policies and Agreements on Human Rights, Oktober 2008, S. 35.

Kenya Women Workers Organisation: The working conditions in the floriculture industry in Kenya. Januar 2008. www.kewwo.or.ke/KEWWO\%20STUDY-Final.pdf (13.02.2010). S. 15.

Dolan/Opondo/Smith (o. Fn. 10) S. 37 f.; US Department of State (o. Fn. 16).

International Federation of Human Rights in cooperation with the Centre for Research on Multinational Cooperation (o. Fn. 17).

Dolan/Opondo/Smith (o. Fn. 10) S. 41.

Marc Engelhardt: Keine rosigen Aussichten, in: Welternährung 38 (2. Quartal 2009), S. 11.

Catherine Riungu: Flower rescue plan as wilting market signals mass closures. The East African 
vermeiden. ${ }^{24}$ Neben Entlassungen wird es dabei sicherlich auch zu Lohnkürzungen kommen. Kurzarbeit kennt das kenianische Arbeitsrecht nicht.

Zusätzlich zum Lohn soll der Arbeitgeber seinem Arbeitnehmer gemäß Artikel 31 Abs. 1 EA auf eigene Kosten eine angemessen Unterkunft am Arbeitsplatz oder in dessen Nähe stellen. In diesem Fall darf er maximal 30 Schillinge vom Lohn einbehalten (Artikel 4 RWO). Alternativ kann er dem Arbeitnehmer eine Summe zahlen für eine angemessene Unterkunft auf eigene Kosten, Artikel 31 Abs. 1 EA. Die meisten mittelgroßen Blumenfirmen bieten ihren Arbeitskräften keine Unterkünfte. Anders dagegen die größeren Unternehmen wie etwa die bereits erwähnten „Sher Agencies“. Sie stellen ihrer Belegschaft Häuser mit einer Fläche von 9 qm zur Verfügung, was für eine Familie sehr beengt ist. In einigen Fällen wird diese Fläche sogar von zwei oder drei Familien bewohnt. ${ }^{25}$ Die Familien werden mit Wasser und Strom versorgt, wobei der Strom nur für Licht, Radio und Fernsehen, nicht aber zum Kochen oder Bügeln genutzt werden darf. Über die Einhaltung wachen unangemeldete Kontrollen. Darüber hinaus bietet diese Firma zahlreiche andere Leistungen für die etwa 16.500 im „Sher-Dorf“ lebenden Menschen: So gibt es eine kostenfreie Krankenschwesternschule, eine Grundschule und eine weiterführende Schule, ein Krankenhaus, eine Mehrzweckhalle und einen Sportplatz. ${ }^{26}$ Das alles ist vorbildlich, schafft aber auch enorme Abhängigkeiten. Die Firma bietet praktisch Leistungen, die eigentlich der Staat zu erbringen hätte, ohne irgendwelche Mitbestimmungsrechte. Arbeiten beide Elternteile für Sher, so ist die Familie der Firma ausgeliefert. Aus diesem Grund werden Sher-Mitarbeiter im Dorf von Außenstehenden auch als Sklavenarbeiter diskreditiert. $^{27}$

\section{Gesundheitsschutz, Sauberkeit und Sicherheit am Arbeitsplatz}

Gesundheitliche Probleme bei den Arbeitern auf den Blumenplantagen sind keine Seltenheit. Dies kann zwei Ursachen haben: Zum einen auf den enormen Einsatz von Pestiziden, zum anderen auf die monotone, bestimmte Körperteile besonders belastende Arbeitsweise. Gesundheitsschutz, Unfallverhütung und Krankheitsvorbeugung sind in verschiedenen Gesetzen geregelt. Der Employment Act und vor allem der Occupational Safety and Health Act 2007 (OSHA) normieren die Pflichten des Arbeitgebers gegenüber dem Arbeitnehmer, um dessen Gesundheit und Integrität bei der Arbeit zu schützen. Darüber hinaus gibt es den

Riungu (o. Fn. 23).

International Federation of Human Rights in cooperation with the Centre for Research on Multinational Cooperation (o. Fn. 17) S. 36.

International Federation of Human Rights in cooperation with the Centre for Research on Multinational Cooperation (o. Fn. 17) S. 34.

27 International Federation of Human Rights in cooperation with the Centre for Research on Multinational Cooperation (o. Fn. 17) S. 36. 
Work Injury Benefit Act 2007 (WIBA), der Rechte und Pflichten im Fall eines Arbeitsunfalls oder einer Berufskrankheit regelt.

Verglichen mit dem internationalen Umfeld zeigen sich Anzeichen einer Überregulierung, deren Details in der Wirklichkeit dieser Arbeitswelt kaum umzusetzen sind. Beispielsweise wird genauestens geregelt, wie ein Arbeitsplatz sauber gehalten werden muss: Schmutz- und Müllanhäufungen müssen täglich von Böden, Arbeitsflächen, Treppen und Übergängen entfernt werden, der Boden muss mindestens einmal pro Woche gewischt oder, sofern das effektiver ist, gefegt werden und alle inneren Wände und Decken müssen je nach Oberfläche einmal im Jahr abgewaschen, einmal in fünf Jahren überstrichen oder lackiert oder einmal im Jahr getüncht werden (Artikel 47 Abs. 1 OSHA). Entsprechend detaillierte Pflichten finden sich zahlreich im OSHA und WIBA, was als Symptom gewertet werden kann, wie weit in der jeweiligen Arbeitswelt Begriffe wie Unfallprävention, Arbeits- und Arbeitsplatzhygiene vielfach für beide Seiten Fremdworte sind. Auch tauchen Strafandrohungen für jegliche Pflichtverstöße insbesondere im OSHA derart zahllos auf, dass Zweifel an der tatsächlichen Verhängung dieser Strafen angebracht sind. Bei einem Verstoß gegen die oben genannten Säuberungspflichten etwa kann eine Strafe von bis zu 300.000 Schillingen (etwa 270 Euro), Freiheitsstrafe bis zu drei Monaten oder beides verhängt werden (Artikel 47 Abs. 2 in Verbindung mit Artikel 109 Abs. 1 OSHA). Gleichwohl vermögen Detailliertheit und Strafandrohungen elementare Rechtsverstöße nicht zu verhindern.

Eine allgemeine Pflicht zur Versorgung der Arbeitnehmer mit einer ausreichenden und sachgerechten Arzneimittelversorgung im Krankheitsfall und, sofern möglich, einer medizinischen Behandlung, enthält Artikel 34 Abs. 1 EA. Dies bezieht sich auf jegliche Art von Krankheit. Der WIBA dagegen nimmt speziell Bezug auf arbeitsbedingte Verletzungen und Krankheiten. Muss ein Arbeitnehmer klinisch versorgt werden, hat der Arbeitgeber - strafbewehrt (Art. 46 Abs. 2 WIBA) - für Hin- und Rücktransport zu sorgen. Ferner muss der Arbeitgeber die medizinischen Ausgaben des Arbeitnehmers bei einem Arbeitsunfall wie Arztbehandlung, Pflegedienst, Arzneimittelkosten usw. tragen (Artikel 47 Abs. 1 WIBA). Verlangt er vom Arbeitnehmer eine Beteiligung an den Kosten, macht er sich gemäß Artikel 50 Abs. 1 WIBA strafbar. Außerdem haftet der Arbeitgeber bei Arbeitsunfällen: Nach Artikel 10 Abs. 2 WIBA muss der Arbeitgeber dem Arbeitnehmer grundsätzlich eine Entschädigung zahlen, wenn dieser sich während der Arbeit verletzt. ${ }^{28}$ Mehr als die Hälfte (56,5\%) der von KEWWO befragten Arbeitnehmer führte an, dass sie in Zusammenhang mit ihrer Arbeit schon unter Gesundheitsproblemen gelitten bzw. Arbeitsunfälle gehabt hätten. ${ }^{29}$ Als Gesundheitsbeschwerden wurden insbesondere geschwollene Beine (19.3\%),

Dies gilt allerdings dann nicht, wenn die Verletzung durch ein vorsätzliches und mutwilliges Fehlverhalten des Arbeitnehmers verursacht wurde (Artikel 10 Abs. 3 WIBA). Diese Ausschlussregelung findet wiederum keine Anwendung bei erheblicher Arbeitsunfähigkeit oder Tod. Als erhebliche Arbeitsunfähigkeit bewertet der WIBA eine dauernde Behinderung von $40 \%$ oder mehr (Artikel 10 Abs. 6 WIBA). 
Rückenschmerzen (17\%) und Erbrechen (3.4\%) genannt. Auch in der Studie des NRI berichteten die Arbeiter über Beschwerden aufgrund des langen Stehens und Bückens und der Belastung mit Pestiziden. ${ }^{30}$ Fast $90 \%$ der Befragten gaben allerdings an, dass sie keine medizinische Unterstützung durch ihren Arbeitgeber erhalten hatten - im Wahrheitsfall ein eindeutiger Verstoß gegen Artikel 47 Abs. 1, 10 Abs. 2 WIBA. Dabei spielen auch die behandelnden Ärzte eine Rolle: Untersuchen sie einen Arbeitnehmer, der einen Arbeitsunfall hatte oder an einer Berufskrankheit leidet, müssen sie innerhalb von 14 Tagen dem Direktor des Dienstes für berufliche Sicherheit und Gesundheit Bericht darüber erstatten (Artikel 48 Abs. 1 WIBA). Die - meist von den Blumenfirmen angestellten - Ärzte verneinen jedoch regelmäßig einen Zusammenhang zwischen der Arbeit auf den Plantagen und den auftretenden gesundheitlichen Problemen, insbesondere den durch Pestizid- und Düngereinsatz verursachten Beschwerden. ${ }^{31}$

Der OSHA verpflichtet den Inhaber einer Arbeitsstätte und den Arbeitgeber, für die Sicherheit, Gesundheit und das Wohlergehen der Beschäftigten zu sorgen (Artikel 6 Abs. 1 OSHA): Es müssen sichere Geräte und Arbeitsverfahren zur Verfügung stehen und die Sicherheit beim Umgang, der Lagerung und dem Transport von Waren und Materialien gewährleistet sein (Artikel 6 Abs. 1 a), b) OSHA). Darüber hinaus ist für die zur Gewährleistung der Sicherheit der Arbeitnehmer notwendige Information und Anweisung zu sorgen (Artikel 6 Abs. 1 c), d) OSHA). Kommt der Arbeitgeber dem nicht nach, drohen Geldstrafe bis zu 500.000 Schillingen (über 4500 Euro), Freiheitsstrafe bis zu sechs Monaten oder beides (Artikel 6 Abs. 7 OSHA). Besondere Anweisungspflichten gelten für gefahrengeneigte Arbeiten, zu denen die Arbeit mit Pestiziden gezählt werden kann. Für solche Arbeiten hat der Arbeitgeber seinem Arbeiter eine spezielle schriftliche Erlaubnis zu erteilen, in der die Tätigkeit, die damit verbundenen Gefahren und die vorzunehmenden Vorsichtsmaßnahmen zu erläutern sind (Artikel 96 Abs. 2 OSHA). Ähnliches wird auch noch einmal in Artikel 99 OSHA betont: Beschäftigte dürfen zu gefahrengeneigter Arbeit nur eingesetzt werden, nachdem sie mit Durchführung und Risiken ausreichend vertraut gemacht sind und von einer erfahrenen Person beaufsichtigt werden. Verstößt der Arbeitgeber hiergegen, drohen ihm Geldstrafe bis zu 200.000 Schillingen, Freiheitsstrafe bis zu sechs Monaten oder beides (Artikel 99 Abs. 6 OSHA). Sehr wichtig für die Arbeit in der Blumenindustrie ist auch die Ausrüstung mit Schutzkleidung, speziell während des Düngens. Nach Artikel 101 OSHA sind Arbeitnehmer, die Nässe oder schädlichen oder angreifenden Substanzen ausgesetzt sind, mit einer adäquaten und wirksamen Schutzkleidung und Hilfsmitteln auszustatten, wie - sofern notwendig - Handschuhen, Schuhen, Atemschutzmasken, Schutzbrillen und Kopfbedeckungen. Allerdings ist diese wesentliche Arbeitgeberpflicht nicht strafbewehrt.

Dolan/Opondo/Smith (o. Fn. 10) S. 46.

31 Food \& Water Watch, The Council of Canadians: Withering under the Assault of International Flower Vendors 2008. www.canadians.org/water/documents/NaivashaReport08.pdf (13.07.2010), S. 3. 
In der Realität sind allerdings sowohl die Aufklärung über mit dem Pestizideinsatz verbundene Gefahren wie auch die Versorgung mit angemessener Schutzkleidung unzureichend. Der größte Teil der Arbeiterinnen ist ungelernt und hat die Schule früh verlassen. Selbst wenn es Vorschriften über Sicherheitsmaßnahmen gibt, werden diese daher häufig nicht verstanden - beispielsweise dürfte es für einen Kenianer, der vorwiegend Swahili spricht, schwierig sein, Sicherheitshinweise über den Umgang mit Pestiziden auf Englisch $\mathrm{zu}$ verstehen, vorausgesetzt er ist überhaupt des Lesens kundig. ${ }^{32}$ Es fehlt somit an einer den Anforderungen des Artikel 96 Abs. 2 OSHA genügenden Gefahrenerläuterung. In der Folge halten die Arbeitnehmer die Sicherheitsvorkehrungen mangels besseren Wissens nicht ein. ${ }^{33}$ Sie empfinden die Schutzkleidung als heiß und unbequem oder können beispielsweise mit Handschuhen nicht im gleichen Tempo arbeiten wie ohne. ${ }^{34}$ Letzteres hat wiederum Auswirkungen auf die Bezahlung, wenn diese sich nicht nach der gearbeiteten Zeit, sondern nach der Anzahl der bearbeiteten Blumenbeete richtet. NRI berichtet von einer Blumenfirma, die ein Bonussystem für die Arbeiter eingeführt hat, die Schutzkleidung tragen. ${ }^{35}$ Dabei dürfte es sich jedoch um eine Ausnahme handeln und es muss wiederum daran erinnert werden, dass die bei der Studie von NRI teilnehmenden Firmen sowieso schon bessere Bedingungen bieten, da sie zertifiziert werden. Doch selbst in diesen Firmen gibt es zahlreiche Klagen der Arbeiter über den Gesundheitsschutz. So müsste eigentlich ein regelmäßiger personeller Wechsel bei den Sprayern durchgeführt werden, um die Auswirkungen der Chemikalien auf den Einzelnen zu verringern. Diese Wechsel werden aber oft nicht durchgeführt. Zudem werden häufig gleichzeitig in einem Gewächshaus Blumen geerntet und Pestizide versprüht und die Wartezeit nach dem Sprühen bis zum erneuten Betreten des Gewächshauses wird oft nicht eingehalten. ${ }^{36}$ Unabhängig von der Frage, ob die Arbeiter die Notwendigkeit von Schutzkleidung verstehen, sind die ihnen zur Verfügung gestellten Materialien in der Regel aber auch unzureichend. Dabei sind Gelegenheitsarbeiter ${ }^{37}$ am wenigsten geschützt: Sie werden meist nicht einmal mit Schutzkleidung ausgerüstet. $^{38}$

Beschwert sich ein Arbeitnehmer über Sicherheits- oder Gesundheitsrisiken, so darf das kein Kündigungsgrund sein, sein Gehalt darf aus diesem Grund nicht gekürzt werden (Artikel 10 Abs. 1 OSHA); er darf auch in keiner (sonstigen) Weise deswegen benachteiligt oder

Die Analphabetenrate in Kenia bei den über 15-Jährigen beträgt etwa 30 Prozent, vgl. United Nations Development Programme (UNDP): Human Development Report 2009, S. 173.

Corporación Cactus: La producción de flores cortadas para la exportación: Un negocio global. Flores en África: El Caso de Kenya. Revista N² 23, S. 2 ff. November 2007.

Dolan/Opondo/Smith (o. Fn. 10) S. 46.

Dolan/Opondo/Smith (o. Fn. 10) S. 46.

Dolan/Opondo/Smith (o. Fn. 10) S: 46.

Siehe zur Lage der Gelegenheitsarbeiter auch unter B. IV.

Dolan/Opondo/Smith (o. Fn. 10) S. 46. 
diskriminiert werden (Artikel 8 As. 1 OSHA). Handelt der Arbeitgeber entgegen diesen Vorschriften, kann dies mit Geld- und Freiheitsstrafe geahndet werden (Artikel 8 Abs. $2^{39}$ bzw. Artikel 10 Abs. 2 in Verbindung mit 109 Abs. 1 OSHA $^{40}$ ). Außerdem kann der Arbeitgeber im Fall der Entlassung oder Diskriminierung dazu verpflichtet werden, dem Arbeitnehmer Schadensersatz zu zahlen und ihn wieder einzustellen (Artikel 8 Abs. 3 OSHA). Eine Schlechterstellung oder eine Entlassung ist auch aus dem Grund unzulässig, dass ein Arbeitnehmer sich wegen einer unmittelbar bevorstehenden und ernsthaften Gefahr für seine Sicherheit oder Gesundheit vom Arbeitsplatz entfernt (Artikel 14 Abs. 2 OSHA). Dieses Recht wird allerdings nicht wahrgenommen, da - sofern sich die Arbeiter dieses Rechts überhaupt bewusst sind - sie den Verlust ihres Arbeitsplatzes befürchten. ${ }^{41}$

Zusammenfassend lässt sich somit sagen, dass eine unzureichende Implementierung der Sicherheits- und Sauberkeitsvorschriften stattfindet. Die verhängten Strafen sind zu gering, als dass sie als Abschreckung dienen könnten. Darüber hinaus gibt es nur 74 Inspekteure, während etwa doppelt so viele für eine effektive Kontrolle benötigt würden. Insbesondere auf den kleinen Blumenplantagen, so kritisieren Gewerkschaften und Nichtregierungsorganisationen, herrschen schlechte Gesundheits- und Sicherheitsstandards. ${ }^{42}$

\section{Die befristet Angestellten}

Ein weiteres grundlegendes Problem in der kenianischen Blumenindustrie, das gleichzeitig großen Einfluss auf die Wahrnehmung und Durchsetzung vieler anderer Arbeitsrechte hat, ist die hohe Anzahl befristeter Verträge. Der Abschluss eines befristeten Vertrags ist durch das Arbeitsrecht an keinerlei Bedingungen geknüpft.

KEWWO kam zu dem Ergebnis, dass über 77\% der von ihnen Befragten nur befristet angestellt waren. ${ }^{43}$ In der Studie des NRI gaben etwa $35 \%$ der Arbeiter an, dass sie nur befristet angestellt seien. ${ }^{44}$ Dabei muss aber beachtet werden, dass die Studie des NRI zum einen aus dem Jahre 2002 stammt; zum anderen, dass nur solche Blumenfarmen teilnahmen, die durch verschiedene Label zertifiziert wurden und daher nicht repräsentativ für die gesamte Blumenindustrie Kenias sind. „Corporación Cactus“, eine Organisation, die Arbeiter auf Blumenplantagen in Kolumbien intensiv unterstützt, sich aber auch mit der Situation auf den Blumenplantagen in Kenia auseinandersetzt, geht davon aus, dass $65 \%$

39

40

41

42

43

44

Mit bis zu 100.000 Schillingen (etwas über 900 Euro), drei Monaten Freiheitsstrafe oder beidem.

Art. 109 Abs. 1 legt eine generelle Strafhöhe von bis zu 300.000 Schillingen (etwa 270 Euro) und Freiheitsstrafe bis zu drei Monaten oder beides für solche Zuwiderhandlungen fest, die keine konkrete Strafandrohung enthalten.

US Department of State (o. Fn. 16).

US Department of State (o. Fn. 16).

Kenya Women Workers Organisation (o. Fn. 18) S. 12.

Dolan/Opondo/Smith (o. Fn. 10) S. 32. 
befristet angestellt sind - und dies in der Regel über viele Jahre hinweg. ${ }^{45}$ Die große Anzahl befristeter Verträge erklärt auch die hohe Fluktuation von Arbeitern. Über $60 \%$ der von KEWWO Befragten gaben an, auf einer Blumenplantage nur ein bis vier Jahre gearbeitet zu haben. ${ }^{46}$

Besonders schlecht unter den befristet Angestellten ist die Situation der Gelegenheitsarbeiter. Nach Artikel 2 EA fallen unter den Begriff des Gelegenheitsarbeiters solche Arbeiter, die am Ende eines jeden Tages bezahlt werden (Tagelöhner) und nicht länger als 24 aufeinander folgende Stunden angestellt sind. Zwar gilt auch für Gelegenheitsarbeiter grundsätzlich das in Artikel 6 Abs. 4 EA normierte Prinzip gleicher Lohn für gleiche Arbeit (,für Arbeit des gleichen Wertes“). Allerdings ergeben sich große Unterschiede aufgrund der Tatsache, dass die Gelegenheitsarbeiter täglich bezahlt werden und damit am Wochenende keinen Lohn erhalten, während Arbeitnehmer mit festen Verträgen monatlich entlohnt werden. Die Zahlung auf Tagesbasis ist den Arbeitgebern gemäß Artikel 18 Abs. 2 a) EA möglich, wonach der Lohn eines Gelegenheitsarbeiters am Ende des Tages fällig ist. Ferner fallen bei allen befristet angestellten Arbeitern zahlreiche Vorteile und Vergünstigungen weg wie Mutterschaftsurlaub, Sozialversicherung, bezahlter Urlaub, Fortzahlung im Krankheitsfall und vieles mehr. ${ }^{47}$ Zwar hat ein Arbeitnehmer, dessen Beschäftigung nach zwei oder mehr (aber weniger als zwölf) aufeinander folgenden Monaten endet, einen Anspruch auf mindestens ein und eindreiviertel Urlaubstage pro Monat (Artikel 28 Abs. 2 b) EA). Über die Umsetzung dieser Vorschrift gibt es aber keine Daten. Die Unsicherheit, die aufgrund des geringen rechtlichen Schutzes unter den Zeitarbeitern vorherrscht, macht den Arbeitern jegliche Zukunftsplanung unmöglich - und dies angesichts der Tatsache, dass die meisten Beschäftigten von der Arbeit auf den Blumenplantagen noch zahlreiche Verwandte ernähren müssen. Aber auch die Wahrnehmung anderer Rechte wird durch die hohe Fluktuation beeinträchtigt. Die ständige Angst vor Entlassung führt dazu, dass insbesondere die befristet angestellten Arbeiter den Launen ihrer Vorgesetzten stärker ausgesetzt sind als Festangestellte. Dies macht Zeitarbeiterinnen, wie sich auch im Folgenden zeigen wird, zu besonders leichten Opfern sexueller Belästigung durch Vorgesetzte. Zudem erschwert der ständige Arbeiterwechsel die Bildung und Effizienz von Gewerkschaften; befristet angestellte Arbeiter sind daher in aller Regel nicht gewerkschaftlich organisiert. ${ }^{48}$

Corporación Cactus (o. Fn. 33) S. 2.

Kenya Women Workers Organisation (o. Fn. 18) S. 14.

Women Working Worldwide: Promoting Women Worker's Rights in African Horticulture. Overview of research into conditions on horticulture farms in Kenya, Zambia, Tanzania and Uganda. Oktober 2007, S. 15.

Women Working Worldwide (o. Fn. 47) S. 15. 


\section{Ein wesentliches Problem: Die Diskriminierung von Arbeiterinnen}

Nicht nur die Verfassung (Artikel 82 Abs. 1, 2) ${ }^{49}$, auch der Employment Act verpflichtet den Arbeitsminister, seine Beamten, das Arbeitsgericht und die Arbeitgeber, für Chancengleichheit zu sorgen und Diskriminierung zu beseitigen (Artikel 5 Abs. 1 und 2 EA). Ausdrücklich genannt wird dabei die Diskriminierung aufgrund von Rasse, Hautfarbe, Geschlecht, Sprache, Religion, politischer oder sonstiger Meinung, Nationalität, ethnischer oder sozialer Abstammung, Behinderung, Schwangerschaft, geistigem Zustand oder HIVErkrankung (Artikel 5 Abs. 3 a) EA). Eine Schlechterstellung darf weder im Hinblick auf die Anwerbung neuer Mitarbeiter, die Ausbildung oder die Beförderung noch in Bezug auf die Arbeitsbedingungen, die Beendigung des Beschäftigungsverhältnisses oder sonstigen auf die Beschäftigung bezogenen Sachverhalten erfolgen. In der Blumenindustrie ist angesichts der hohen Beschäftigungsrate von Frauen insbesondere die Diskriminierung aufgrund des Geschlechts ein wesentliches Problem. Im kenianischen Recht gilt gemäß Artikel 6 Abs. 4 EA das Prinzip gleicher Lohn für gleiche Arbeit, welches formell in der Blumenindustrie auch eingehalten wird. ${ }^{50}$ Frauen verdienen im Durchschnitt faktisch aber weniger als Männer, was an der Art der Arbeit liegt, die sie durchführen: Männer arbeiten in der Regel in der Bewässerung, der Instandhaltung oder in den Kühlhäusern, als „Sprüher“ in den Gewächshäusern oder als Sicherheitspersonal, was als Tätigkeiten unter "schweren Bedingungen" betrachtet wird. Frauen dagegen sind meist mit Aufgaben wie dem Pflücken, Sortieren und Packen der Blumen betraut - Tätigkeiten, die als einfach eingestuft werden, unabhängig von den harten körperlichen Bedingungen, die gerade beim Pflücken an die Frauen gestellt werden. ${ }^{51}$ Darüber hinaus werden Männer mehr befördert und besser fortgebildet. Über $80 \%$ der von KEWWO befragten Arbeiter gaben an, dass bei Fortbildungsmaßnahmen Männer bevorzugt würden. ${ }^{52}$ Dies wiederum ist auf die Arbeitsverteilung zurückzuführen, da Fortbildungen eben vorwiegend für die von Männern ausgeführten oben genannten Tätigkeiten angeboten werden. ${ }^{53}$

Ein weiteres großes Problem in diesem Zusammenhang stellt die sexuelle Belästigung von Arbeiterinnen durch Vorgesetzte und Kollegen dar. Dies ist gesetzlich zwar in aller Deutlichkeit untersagt, aber auf den Blumenplantagen haben diese Regelungen noch keine Implementierung erfahren. Sexuelle Belästigung einer Arbeitnehmerin liegt dann vor, wenn

Die Verfassung sieht allerdings Ausnahmen des Diskriminierungsverbots beispielsweise in den Bereichen der Ehe, der Scheidung und des Erbrechts oder des Gewohnheitsrechts von Stämmen vor (Artikel 82 IV). Der neue Verfassungsentwurf enthält dagegen ein ausnahmsloses Gleichbehandlungsgebot, s. dazu u. E.

Dolan/Opondo/Smith (o. Fn. 10) S. 41.

Dolan/Opondo/Smith (o. Fn. 10) S. $41 \mathrm{f}$.

52

53

Kenya Women Workers Organisation (o. Fn. 18) S. 22.

Dolan/Opondo/Smith (o. Fn. 10) S. 40. 
der Arbeitgeber, einer seiner Vertreter oder ein Kollege eine Arbeitnehmerin ${ }^{54}$ direkt oder indirekt zum Geschlechtsverkehr oder sexuellen Kontakt auffordert (Artikel 6 Abs. 1 EA). Ferner fällt darunter die Aufforderung zu jeder Art von sexueller Aktivität, in deren Gegenzug eine bevorzugte Behandlung bei der Arbeit versprochen oder bei deren Verweigerung mit beruflichen Nachteilen gedroht wird (Artikel 6 Abs. 1 a) EA). Auch verbale oder schriftliche sexuelle Belästigungen können den Tatbestand erfüllen (Artikel 6 Abs. 1 b) EA). Allerdings regelt der Employment Act keine Folgen im Fall einer sexuellen Belästigung am Arbeitsplatz.

Ein Vorgehen gegen sexuelle Belästigung ist jedoch auf der Grundlage des Sexual Offences Act 2006 (SOA) möglich. Nach Artikel 23 Abs. 1 SOA macht sich eine Autoritätsperson strafbar, die einer anderen Person beharrlich sexuelle Annäherungsversuche macht. ${ }^{55}$ Notwendig für eine Verurteilung ist allerdings der Nachweis, dass der Täter beabsichtigt hatte, die Reaktion des Opfers als Grundlage für die Entscheidung über die Einstellung oder das berufliche Fortkommen des Opfers heranzuziehen (Artikel 23 Abs. 2 a) SOA). Ausreichend ist aber auch, dass die Annäherungsversuche in der Absicht stattfanden, in die Arbeit des Opfers störend einzugreifen oder eine anstößige Arbeitsatmosphäre zu schaffen (Artikel 23 Abs. 2 b) SOA). Darüber hinaus legt der Employment Act den Arbeitgebern, die 20 und mehr Personen beschäftigen, die Pflicht auf, nach Beratung mit den Arbeitnehmern und ihren Vertretern (sofern vorhanden) eine grundsätzliche Stellungnahme zu sexueller Belästigung abzugeben (Artikel 6 Abs. 3 EA). Darin soll unter anderem ein Verfahren angegeben werden, über das eine Arbeitnehmerin den Arbeitgeber von sexueller Belästigung benachrichtigen kann, ohne dass sie namentlich bekannt wird.

Schon die ausführlichen gesetzlichen Regelungen zeigen, dass sexuelle Belästigung am Arbeitsplatz ein großes Problem in Kenia darstellt. Auch auf den Blumenfarmen ist sie sehr verbreitet. Das NRI beobachtete bei seiner Studie, dass an allen untersuchten Blumenfarmen sexuelle Belästigung vorkam. In der Regel war das Management jedoch der Auffassung, dass auf seiner Plantage sexuelle Belästigung kein Problem sei, und selbst dort, wo man entsprechende Vorkommnisse einräumte, wurde der Ursprung des Problems in der kenianischen Kultur gesehen und nicht als Aufgabe der Verantwortung durch die Firma. ${ }^{56}$ Bei KEWWO gaben $14 \%$ der befragten Arbeiter an, schon um sexuelle Gefälligkeiten gebeten worden zu sein ${ }^{57}$, wobei beachtet werden muss, dass knapp 33\% der Befrag-

Das Gesetz spricht zwar von „employer“ und ,employee“ und umfasst damit theoretisch natürlich auch Arbeitgeberinnen und männliche Arbeitnehmer. Da aber die Belästigung von Frauen im Vordergrund steht - und auch nur von dieser berichtet wird - beschränke ich mich im Folgenden allein auf die Arbeiterinnen.

Dieses Verhalten kann mit einer Geldstrafe von bis zu 100.000 Schillingen (etwas mehr als 900 Euro) oder mit Freiheitsstrafe nicht unter drei Jahren oder beidem geahndet werden (Artikel 23 Abs. 1 SOA).

Dolan/Opondo/Smith (o. Fn. 10) S. 39. 
ten Männer waren und der Prozentsatz unter den Arbeiterinnen damit noch höher liegt. Zum größten Teil (über 63\%) handelte es sich bei den Tätern um die Aufseher der Frauen, als zweite Gruppe folgten männliche Kollegen. ${ }^{58}$ Von den Arbeiterinnen, die sexuelle Belästigung schon erfahren hatten, gaben $45 \%$ an, bereits mehr als 14 mal nach sexuellen Diensten gefragt worden zu sein. Meistens weigerten sich die Frauen ihrem Vorbringen nach allerdings, den Forderungen nachzukommen. Von denen, die angaben, dass es zu sexuellen Handlungen gekommen war, wurden $42 \%$ zum Geschlechtsverkehr gezwungen. ${ }^{59}$ Opfer sind insbesondere die befristet angestellten Arbeiterinnen. Sie sind ,erpressbar"; im Gegenzug für sexuelle Dienste werden ihnen der Erhalt des Arbeitsplatzes, Zusatzleistungen oder eine Beförderung versprochen. ${ }^{60}$ Es gibt - entgegen beispielsweise Art. 6 Abs. 3 EA - keine Einrichtungen oder Verfahren innerhalb oder außerhalb der Blumenfirmen für ein Vorgehen gegen sexuelle Belästigung. Da die Täter, wie schon erwähnt, meist die Vorgesetzten oder Aufseher sind, können sie sich an diese schon einmal nicht wenden. Die Polizei hat nicht das Vertrauen der Frauen und ist für den objektiven Umgang mit dieser Thematik nicht ausgebildet. ${ }^{61}$

Eine Diskriminierung von Arbeiterinnen erfolgt ferner entgegen dem Verbot der Ungleichbehandlung aus Gründen des Geschlechts in Artikel 5 Abs. 3 a) EA bei Einstellung und Entlassung von Arbeiterinnen aufgrund von Schwangerschaft. So werden zum Beispiel Schwangerschaftstests vor der Einstellung auf Plantagen durchgeführt. ${ }^{62}$ Außerdem berichten einige Blumenfrauen, dass nicht fest angestellte Arbeiterinnen vor dem Abschluss eines dauerhaften Vertrags einen Schwangerschaftstest durchführen lassen müssen und bei einem positiven Ergebnis der Vertragsabschluss verweigert wird. ${ }^{63}$ Zudem kommen Kündigungen aufgrund einer Schwangerschaft oder eines Antrags auf Mutterschaftsurlaub ${ }^{64}$ vor. $^{65}$ Dies verstößt nicht nur gegen das allgemeine Diskriminierungsverbot des Artikels 5 Abs. 3 a) EA, sondern auch gegen den speziellen Kündigungsschutz, den Mütter erfahren. Nach Artikel 45 Abs. 1 EA darf eine Auflösung des Beschäftigungsverhältnisses nicht unfair erfolgen. Ist aber die Schwangerschaft oder ein damit zusammenhängender Grund Motiv für die Kündigung, so stellt dies eine unfaire und damit unzulässige Kündigung dar (Art. 45 Abs. 4 a), 46 a) EA).

Kenya Women Workers Organisation (o. Fn. 18) S. 25.

Kenya Women Workers Organisation (o. Fn. 18) S. 25.

Dolan/Opondo/Smith (o. Fn. 10) S. 39. Kenya Women Workers Organisation (o. Fn. 18) S. 6, 25.

Kenya Women Workers Organisation (o. Fn. 18) S. 6.

Dolan/Opondo/Smith (o. Fn. 10) S. 41.

Dolan/Opondo/Smith (o. Fn. 10) S. 41.

Gemäß Artikel 29 Abs. 1 EA steht einer Arbeiterin ein dreimonatiger Mutterschaftsurlaub mit voller Bezahlung nach zu, s.u. C.

Women Working Worldwide (o. Fn. 47) S. 20. 
Leider gibt es meinen Recherchen zufolge weder konkrete Daten über die Diskriminierung von schwangeren Frauen noch über die Folgen einer unzulässigen Entlassung. Doch selbst für den Fall, dass den Frauen ihre Rechte bewusst sind und sie ein Vorgehen gegen die Entlassung in Erwägung ziehen, dürfte dies schon aus Beweisgründen kaum erfolgreich sein: Dem kenianischen Recht fehlt beispielsweise eine Vermutungsregel, nach der angenommen wird, dass die Schwangerschaft Motiv für die Kündigung war, wenn die Kündigung innerhalb eines bestimmten Zeitraums vor oder nach der Geburt erfolgte. Ohne eine solche Regelung dürfte es einer Arbeiterin kaum möglich sein, einen entsprechenden Nachweis zu erbringen. Das kenianische Recht räumt einer Arbeiterin ferner einen dreimonatigen Mutterschaftsurlaub mit voller Bezahlung ein (Artikel 29 Abs. 1 EA). Nach dem Mutterschaftsurlaub hat sie das Recht, wieder an ihren alten Arbeitsplatz oder einen anderen angemessenen Arbeitsplatz zu nicht ungünstigeren Bedingungen als bisher zurückzukehren (Artikel 29 Abs. 2 EA). Voraussetzung ist, dass die Arbeiterin ihren Arbeitgeber grundsätzlich nicht weniger als sieben Tage zuvor über den geplanten Mutterschaftsurlaub informiert und auf Verlangen des Arbeitgebers ein Zertifikat eines Arztes oder einer Hebamme vorlegt, das ihre Schwangerschaft bestätigt (Artikel 28 Abs. 4 - 6 EA). Über die tatsächliche Gewährung von Mutterschaftsurlaub gibt es keine konkreten Erhebungen. Es wird jedoch berichtet, dass der Mutterschaftsurlaub häufig gar nicht oder nur unbezahlt gewährt wird. ${ }^{66}$ Ferner verstecken Arbeiterinnen, sofern möglich, ihre Schwangerschaft oder treiben aus Angst vor einem Arbeitsplatzverlust ab und fordern daher ihr Recht auf Mutterschaftsurlaub gar nicht erst ein. ${ }^{67}$

Die verschiedenen Beispiele zur alltäglichen Diskriminierung von Arbeiterinnen auf den Blumenplantagen zeigen deutlich, dass zu einer Förderung von Frauen eine gesetzliche Gleichstellung allein bei weitem nicht ausreicht. Vielmehr trifft die von Riedel/Pollak schon 1986 festgestellte Beobachtung zu, dass in vielen Ländern der rechtliche Regelungsstand der Entwicklung der Gesellschaft voraus ist ${ }^{68}$ - so auch bei den Rechten der Arbeiterinnen in Kenia. Die deutliche Rollenverteilung in der Blumenindustrie basiert auf dem in der kenianischen Gesellschaft vorherrschenden, traditionellen Frauenbild: Männer werden als Führer betrachtet, Frauen dagegen wird eine Schwäche zugeschrieben, aufgrund derer sie nicht als Führer geeignet sind. ${ }^{69}$ Auch unter den Arbeitern auf den Blumenplantagen wird die Geschlechterverteilung als natürliche Folge der sozialen Normen und biologischen Unterschiede betrachtet und daher hingenommen. ${ }^{70}$ Erforderlich wären daher eine bessere Ausbildung von Frauen und eine Aufklärung über ihre Rechte, um dauerhaft die Rolle der

Ethical Trading Initiative: Addressing labour practices on Kenyan flower farms. London 2005. www.gg.rhul.ac.uk/kenya/ETIrept-KenyaFlowers2005.pdf (13.07.2010). S. 10.

Dolan/Opondo/Smith (o. Fn. 10) S. 10.

Jürgen Riedel / Christian Pollak: Recht und Entwicklung. Rechtliche Rahmenbedingungen privatwirtschaftlicher Tätigkeit in Entwicklungsländern. München/Köln/London 1986. S. 94. 
Frau in der kenianischen Gesellschaft zu verbessern. Dass dies aber ein langwieriger Prozess ist, an dem die ganze Gesellschaft mitwirken muss, lässt sich auch in Europa noch deutlich erkennen. Speziell in der Blumenindustrie könnte sicherlich schon eine stärkere Beförderung von Frauen einiges bewirken, vor allem in Bezug auf sexuelle Belästigungen und den Umgang mit schwangeren Arbeiterinnen.

\section{Das Kollektivarbeitsrecht und die tatsächliche Situation der Gewerkschaften}

Der Labour Relations Act (LRA) definiert eine Gewerkschaft als eine Vereinigung von Arbeitnehmern mit dem vorrangigen Ziel, die Beziehungen zwischen Arbeitnehmern und Arbeitgebern oder Arbeitgebervereinigungen zu regeln (Artikel 2 LRA). Das Gesetz enthält - wie die Verfassung in Art. 80 - weitreichende Regelungen, um die Aktivitäten von Gewerkschaften zu schützen: So normiert Artikel 4 Abs. 1 LRA das Recht jeden Arbeitnehmers, eine Gewerkschaft zu gründen, Mitglied einer Gewerkschaft zu werden und aus dieser wieder auszutreten. Arbeitnehmer dürfen nicht aus dem Grund diskriminiert werden, dass sie ein ihnen durch den LRA eingeräumtes Recht wahrnehmen (Artikel 5 Abs. 1 LRA). Insbesondere dürfen sie nicht bedroht werden, um dadurch an der Gewerkschaftsmitgliedschaft oder der Ausübung eines damit zusammenhängenden Rechts gehindert zu werden, und eine Kündigung oder eine sonstige Schlechterstellung wegen des jetzigen oder früheren Engagements in einer Gewerkschaft ist unzulässig (Artikel 5 Abs. 2 LRA). Einem Arbeitnehmer darf ferner kein Vorteil dafür versprochen werden, dass er eines der im LRA genannten Rechte nicht wahrnimmt (Artikel 5 Abs. 3 LRA). Auch das Streikrecht ist im kenianischen Arbeitsrecht verankert. Eine Person darf nach Artikel 76 LRA dann an einem Streik teilnehmen, wenn eine Arbeitsstreitigkeit vorliegt, die die Arbeitsbedingungen oder die Anerkennung einer Gewerkschaft betrifft, diese Streitigkeit durch Vermittlung nach dem LRA oder einem in einem Tarifvertrag vorgesehenen Schlichtungsverfahren nicht gelöst werden konnte und das Ministerium und die anderen betroffenen Parteien durch die Gewerkschaft sieben Tage vor Beginn des Streiks davon in Kenntnis gesetzt werden. Der Arbeitgeber kann in einem dringenden Fall gegen den Streik beim Handelsgericht vorgehen, wenn der Streik nach dem LRA nicht zulässig ist - etwa weil der Streitgegenstand schon durch einen Tarifvertrag geregelt worden ist (Artikel 78 Abs. 1 b) LRA) - oder wenn die Gewerkschaft nicht mit gutem Glauben, die Streitigkeit aus dem Weg zu räumen, an den Verhandlungen teilgenommen hat (Artikel 77 Abs. 1 LRA).

Ist der Streik aber rechtmäßig, so ist es dem Arbeitgeber untersagt, jegliche disziplinarische Maßnahme oder die Kündigung gegen einen Arbeitnehmer auszusprechen, der am Streik oder bei anderen damit in Zusammenhang stehenden Tätigkeiten teilgenommen hat (Artikel 79 Abs. 3 LRA). Allerdings muss der Arbeitgeber dem Arbeitnehmer während des Streiks keinen Lohn zahlen (Artikel 79 Abs. 6 LRA).

An der Wahrnehmung des Rechts auf Gewerkschaftsgründung und Streik mangelt es in mehrfacher Hinsicht. Eine effiziente gewerkschaftliche Vertretung auf den Blumenplantagen ist rar, wobei es keine verlässlichen Zahlen über die gewerkschaftliche Organisation in 
der kenianischen Blumenindustrie gibt. Immerhin mehr als die Hälfte (56.6\%) der von KEWWO Befragten gaben an, Mitglied in einer Gewerkschaft zu sein, wobei aber beachtet werden muss, dass die an der Studie teilnehmenden Firmen solche waren, die verschiedene Zertifikate für fairen Handel erhalten. In der vom NRI durchgeführten Studie ergab sich dagegen ein anderes Bild: Etwa die Hälfte der befragten Blumenfirmen hatte eine gewerkschaftliche Vertretung, aber nur in drei Fällen waren mehr als 50 Prozent der Arbeitnehmerschaft auch Mitglied dieser Gewerkschaft. ${ }^{71}$ Diese geringen Mitgliedschaftszahlen haben mehrere Ursachen. Eine große Rolle spielt die Ineffizienz der kenianischen Gewerkschaften. So waren fast $70 \%$ der von KEWWO Befragten der Meinung, dass Gewerkschaften ihre Interessen nicht angemessen vertreten würden. ${ }^{72}$ Auch die Teilnehmer der Studie des NRI berichteten von ihrer Unzufriedenheit; beispielsweise hätten sie sich bei einer Gewerkschaft registriert und danach nicht gewusst, wofür sich die Gewerkschaft engagiere, für die sie Beiträge zahlten. ${ }^{73}$ Von der Vertretung der Friedrich-Ebert-Stiftung in Kenia erhielt ich die Auskunft, sie arbeite nicht mit der für die Blumenindustrie zuständigen Gewerkschaft zusammen, da ihres Erachtens von deren Seite aus kein ernsthaftes Interesse an einer inhaltlichen Arbeit bestehe.

Ein weiteres großes Hindernis für eine stärkere gewerkschaftliche Organisation ist ferner die hohe Anzahl der befristeten Angestellten. Diese sind in aller Regel nicht gewerkschaftlich organisiert. ${ }^{74}$ Zwar steht ihnen dieses Recht grundsätzlich gemäß Artikel 4 LRA wie jedem anderen Arbeitnehmer zu; die Wahrnehmung ist allerdings schon aufgrund der tatsächlichen Umstände - z.B. den ständigen Arbeitsplatzwechseln - so gut wie unmöglich. Sie werden daher auch schon bei der Rekrutierung überhaupt nicht berücksichtigt. ${ }^{75}$ Darüber hinaus werden gewerkschaftliche Aktivitäten, beispielsweise durch Einschüchterungen, vom Management, unterdrückt. ${ }^{76}$ Diese Einschüchterungen sind allerdings auch deshalb so effizient, weil die Arbeitnehmer ihre Rechte in der Regel nicht kennen. So gaben einige Arbeiter - offensichtlich in Unkenntnis der Tatsache, dass dies gemäß Artikel 5 LRA unzulässig ist und nach Artikel 82 Abs. 2 LRA mit einer Geldstrafe von 40.000 Schillingen (etwa 357 Euro) geahndet werden kann - an, das Management würde eine gewerkschaftliche Organisation verbieten. Nahezu alle derjenigen von KEWWO Befragten, die nicht Mitglied einer Gewerkschaft waren, führten an, sie befürchteten negative Konsequenzen im

Anne Tallontire / Catherine Dolan / Sally Smith / Stephanie Barrientos: Reaching the marginalised? Gender value chains and ethical trade in African horticulture. Development in Practice. Volume 15 (2005), 559 (567).

Kenya Women Workers Organisation (o. Fn. 18) S. 23.

73

Dolan/Opondo/Smith (o. Fn. 10) S. 43.

Women Working Worldwide (o. Fn. 47) S. 15.

Dolan/Opondo/Smith (o. Fn. 10) S. 44.

76

Dolan/Opondo/Smith (o. Fn. 10) S. 43. 
Fall eines Gewerkschaftsbeitritts. ${ }^{77}$ In der Studie des NRI waren einige Arbeiter der Auffassung, ihnen würde gekündigt, wenn sie sich in einer Gewerkschaft engagierten. ${ }^{78}$ Jedoch gibt es nur in einer Studie Hinweise darauf, dass aus diesem Grund in der Blumenindustrie tatsächlich Kündigungen ausgesprochen werden. ${ }^{79}$

Neben Gewerkschaften gibt es auf einigen Blumenfarmen so genannte ,workers' committees“. Dabei handelt es sich um innerbetriebliche Gremien, die aus gewählten Vertretern des Personals bestehen und das Mandat haben, in deren Namen mit dem Arbeitgeber zu verhandeln. ${ }^{80}$ Dieses basiert allerdings auf keiner gesetzlichen Regelung, sondern wird aufgrund eines Verhaltenskodexes eingerichtet, zu dessen Einhaltung sich das betreffende Unternehmen zur Erlangung eines Zertifikats verpflichtet hat. Von den Arbeitern wird deren Arbeit als effizienter für die Durchsetzung der Anliegen der Arbeitnehmer beurteilt als die Arbeit der Gewerkschaften. Dies kann zum einen natürlich auf den Mangel an Gewerkschaften zurückgeführt werden; zum anderen auf die ineffektive Arbeit dort, wo Gewerkschaften existieren. ${ }^{81}$ Aber auch die Komitees werden nicht als optimale Interessenvertretung eingestuft. So gaben nur 33\% an, dass deren Arbeit zu einer Verbesserung ihrer Situation geführt habe, während der größte Teil anführte, dass aufgrund von Untätigkeit oder Laxheit der Komitees ihre Situation sich nicht verändert habe. Berichtet wurde allerdings auch von Fällen aktiver Komitees, die dann schnell durch das Management zur Ruhe gebracht wurden, etwa durch Beförderungsangebote. ${ }^{82}$ Letztlich können diese Gremien keine Gewerkschaft ersetzen. Dazu hängen sie zu sehr von dem Willen des Unternehmens zur Erlangung einer Zertifizierung und der Effizienz der Kontrolle der Zertifizierungsvoraussetzungen $\mathrm{ab}$.

\section{E. Schlussbemerkung}

Dies ist nur ein kurzer Überblick über die Diskrepanz zwischen Gesetzeslage und Gesetzesumsetzung in der kenianischen Blumenindustrie. Noch viele weitere Punkte könnten behandelt werden, etwa das Überschreiten der zulässigen Anzahl von Überstunden, Verstöße gegen sozialrechtliche Vorschriften oder die massive Verschmutzung von Wasser durch die Blumenindustrie, wodurch Fischer und Anwohner ihre Lebensgrundlagen verlieren. Überall jedoch kommt man zu dem Ergebnis, dass selbst wenn es ausreichende Gesetze gibt, diese nicht implementiert werden. Die Gründe dafür, die von Korruption, mangelnder

Kenya Women Workers Organisation (o. Fn. 18) S. 23.

Dolan/Opondo/Smith (o. Fn. 10) S. 43.

International Federation of Human Rights in cooperation with the Centre for Research on Multinational Cooperation (o. Fn. 17) S. 38 f.

Dies scheint insbesondere auf den Blumenplantagen der Fall zu sein, die zertifiziert werden. Dolan/Opondo/Smith (o. Fn. 10) S. 43.

Dolan/Opondo/Smith (o. Fn. 10) S. 44.

Dolan/Opondo/Smith (o. Fn. 10) S. 44. 
Kenntnis von Rechten bis zu fehlender örtlicher und finanzieller Zugänglichkeit zu Gerichten reichen, sind vielfältig. Gerade im Arbeitsrecht wirkt sich eine fehlende Implementierung aber enorm auf das Leben der Menschen aus: Dieser Rechtsbereich betrifft die existentiellen Lebensgrundlagen für die Masse der Bevölkerung (meist) während der gesamten Lebensdauer.

Hoffnung macht die neue Verfassung, für die sich nach dem Parlament auch die Bevölkerung Kenias im August 2010 in einem Referendum ausgesprochen hat. Sie enthält erstmals einen langen und fortschrittlichen Grundrechtekatalog (,Bill of Rights“) in den Artikel 26 - 51. Die Ungleichbehandlung der Geschlechter wird in allen Artikeln aufgehoben; ein Gleichbehandlungsgebot und ein Diskriminierungsverbot sind ausdrücklich enthalten (Artikel 27). Ferner wird das Recht auf faire Arbeitspraktiken, eine faire Bezahlung, angemessene Arbeitsbedingungen und das Recht zur Gründung von Gewerkschaften und zur Teilnahme an gewerkschaftlichen Aktivitäten festgelegt (Artikel 41). Erstmals hat nunmehr der Gesetzgeber den Auftrag, selbständige Arbeitsgerichte zu schaffen (Art. 162 Abs. 2, lit.a). Es bleibt abzuwarten, wie weit sich die neue Verfassung auf die Arbeitsbedingungen in der Blumenindustrie auswirkt. Solange die europäischen und amerikanischen Blumenhändler von ihren afrikanischen und lateinamerikanischen Handelspartnern Niedrigstpreise und eine möglichst hohe Flexibilität erwarten, dürfte eine Besserung in naher Zukunft kaum zu erwarten sein. 